bility of DNase I. BioTechniques 13:64-66.

2.Cusi, M.G., M. Valassina and P.E. Valensin. 1994. Comparison of M-MLV reverse transcriptase and Tth polymerase activity in RTPCR of samples with low virus burden. BioTechniques 17:1034-1036.

3.Fiorenza, M.T. and F. Mangia. 1998. Quantitative RT-PCR amplification of RNA in single mouse oocytes and preimplantation embryos. BioTechniques 24:618-623.

4.Huang, Z., M.J. Fasco and L.S. Kaminsky. 1996. Optimization of DNase I removal of contaminating DNA from RNA for use in quantitative RNA-PCR. BioTechniques 20:1012-1020.

5.Kunitz, M. 1950. Crystalline desoxyribonuclease: I. Isolation and general propertiesSpectrophotometric method for the measurement of deoxyribonuclease activity. J. Gen. Physiol. 33:349-362.

6.Mallet, F., G. Oriol, C. Mary, B. Verrier and B. Mandrand. 1995. Continuous RT-PCR using AMV-RT and Taq DNA polymerase: characterization and comparison to uncoupled procedures. BioTechniques 18:678-687.

7.Rand, K.H. and H. Houck. 1990. Taq polymerase contains bacterial DNA of unknown origin. Mol. Cell. Probes 4:445-450.

8.Rochelle, P.A., A.J. Weightman and J.C. Fry. 1992. DNase I treatment of Taq DNA polymerase for complete PCR decontamination. BioTechniques 13:520.

9.Schmidt, T.M., B. Pace and N.R. Pace. 1991. Detection of DNA contamination in Taq polymerase. BioTechniques 11:176-177.

10.Zimmerman, S.B. and N.F. Coleman. 1971 Pancreatic deoxyribonuclease-The role of dimerization in the reversible thermal inactivation at acid pH. J. Biol. Chem. 246:309-317.

Address correspondence to Ken-ichi Hanaki, Department of Bacteriology, Faculty of Medicine, The University of Tokyo, 7-3-1 Hongo, Bunkyo-ku, Tokyo 113-0033, Japan.e-mail:hanakims@d1.dion.ne.jp

Received 30 June 1999; accepted 21 January 2000.

K. Hanaki, H. Nakatake, $K$. Yamamoto $^{1}$, T. Odawara ${ }^{2}$ and H. Yoshikura ${ }^{2}$

The Chemo-Sero Therapeutic

Research Institute

Kyokushi, Kikuchi-gun

Kumamoto

${ }^{1}$ Laboratory of Clinical

Microbiology and Immunology

Bun'inn Hospital

of University of Tokyo

Tokyo

${ }^{2}$ National Institute

of Infectious Diseases

Tokyo, Japan

\section{Trypan Blue as a Slow Migrating Dye for SSCP Detection in Polyacrylamide Gel Electrophoresis}

BioTechniques 29:42-44 (July 2000)

Bromophenol blue (BPB) and xylene cyanol (XC) are classic dyes used for DNA and RNA gel electrophoresis as indicators of migration distance. Since their migration corresponds to less than $500 \mathrm{bp}$ in polyacrylamide gels, they often run off the gel when we tried to investigate the high molecular weight area of the gel. SSCP with silver staining (SSCP-SS) is an easy, nonradioisotopic method for screening point mutations in known regions of genomic DNA derived from pathology archives, fresh tissues and peripheral blood cells (1). It uses less expensive equipment, such as ordinary $14 \times 14-\mathrm{cm}$ slab gel electrophoresis. It has become one of the best methods for screening the mutation and polymorphism in genetics and molecular epidemiology in the modestly equipped laboratories found often in suburban hospitals. In this technique, single strands of DNA

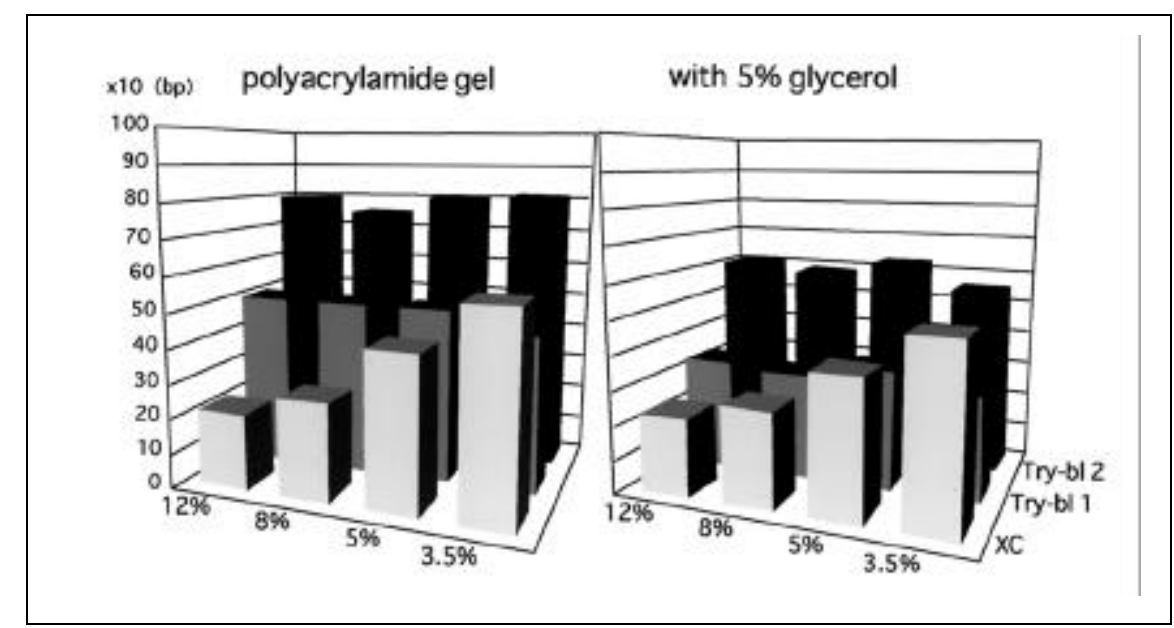

Figure 1. Relative migration distance of three dyes in polyacrylamide gel with and without $5 \%$ glycerol. Basepair was estimated and adjusted by $\phi \mathrm{X}$ 174/HaeIII.

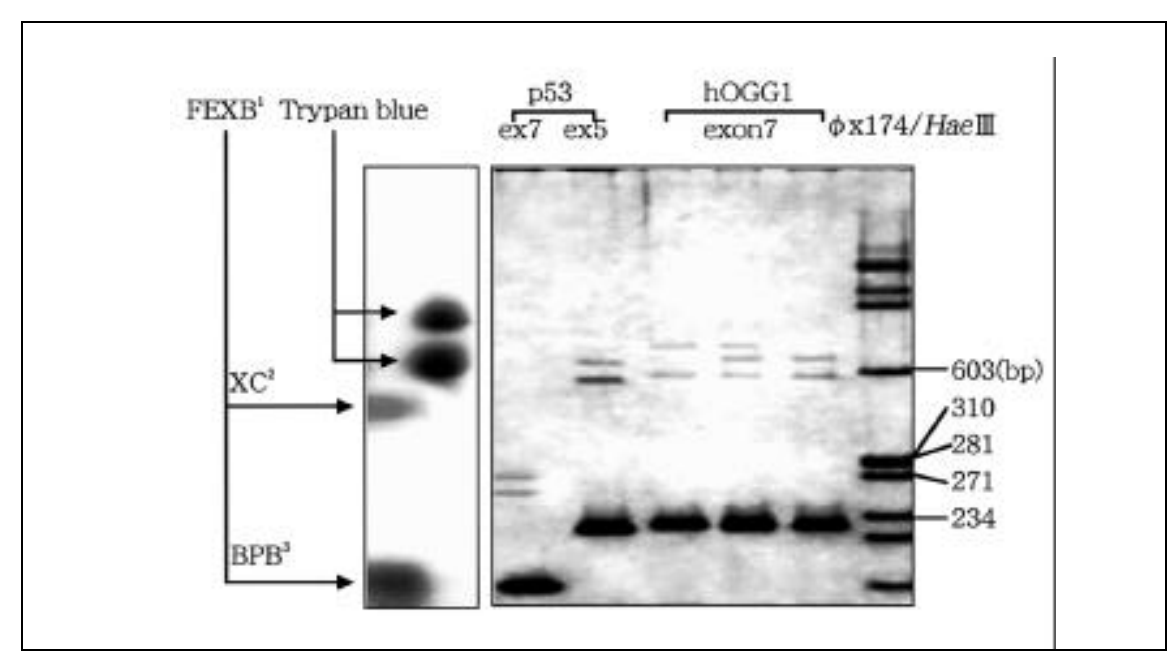

Figure 2. Relative migration distance of BPB, XC (the left lane) and Trypan blue (lane 2). SSCP analysis after visualization using a silver staining kit (Wako, Osaka, Japan) showing p53 exons 7 and 5 and three genotypes of Ser326Cys polymorphism of hOGG1 (exon 7) (2). The rightmost lane is a size marker $\phi X$ 174/HaeIII (Toyobo, Osaka, Japan). 
migrate much more slowly than the dsDNA, and a minute difference in migration distance caused by the conformation change of single strands is visualized by silver staining. Because single strands migrate slower, the area visualized is in the higher molecular weight range of gel electrophoresis. This requires longer electrophoresis times, frequently until $\mathrm{BPB}$ and $\mathrm{XC}$ run off the gel.

We screened the commonly used, less expensive dyes of smaller negative charge and larger molecular weight, looking for a migration indicator useful for long electrophoresis times and visually detectable without fluorescence. Trypan blue, commonly used for vital staining, was discovered to migrate much more slowly than XC and to generate two bands visible in ordinary TBE buffers. In Figure 1, the migration distance of this dye is compared to that of $\mathrm{XC}$ and BPB (both from Katayama Chemicals, Nagoya, Japan) in polyacrylamide gels of different acrylamide concentration with and without glycerol. We usually put this dye in a blank gel as a migration indicator to approximate when to stop the electrophoresis used to detect polymorphism in genomic DNA (Figure 2). In our experience, this dye does not seem to harm the electrophoresis pattern of undigested and digested genomic DNAs.

\section{REFERENCES}

1.Dobashi, Y., H. Sugimura, A. Sato, T. Hirabayashi, H. Kanda, T. Kitagawa, N. Kawaguchi, T. Imamura and R. Machinami. 1993. Possible association of p53 overexpression and mutation with high-grade chondrosarcoma. Diagn. Mol. Pathol. 2:257-263.

2.Sugimura, H., T. Kohno, K. Wakai, K. Nagura, K. Genka, H. Igarashi, B.J. Morris, S. Baba et al. 1999. hOGG1 Ser326Cys polymorphism and lung cancer susceptibility. Cancer Epidemiol. Biomarkers Prev. 8:669-674.

Address correspondence to Dr. Haruhiko Sugimura, The First Depatment of Pathology, Hamamatsu University School of Medicine, 3600 Handa-cho, Hamamatsu, Shizuoka, 431-31, Japan. e-mail: hsugimur @hama-med.ac.jp

Received 17 January 2000; accepted 13 March 2000.

\section{Hisaki Igarashi, Kiyoko Nagura and Haruhiko Sugimura \\ Hamamatsu University \\ School of Medicine \\ Hamamatsu, Japan}

\section{CEL I Enzymatic Mutation Detection Assay}

\section{BioTechniques 29:44-48 (July 2000)}

Mutations in key genes are a common basis for cancer $(1,3)$. In addition, many genes are polymorphic among people, and some polymorphisms can lead to biological changes $(2,4)$. Several procedures have been devised for the detection of mutations and polymorphisms in genes, but not all of them are reliable, economical or easy to use. The method developed in this laboratory, CEL I mutation detection, is simple and dependable. CEL I is an endonuclease isolated from celery (6), and is the first known endonuclease to have high specificity for insertions, deletions and base-substitution mismatches. The assay uses fluorescently or radioactively labeled nucleotides for fragment detection. Briefly, PCR is used to amplify the normal and mutant alleles of the target sequence. The PCR primers have a 5 ' fluorescent tag, FAM (6-carboxyfluorescein) on the forward primer and TET $\left(4,7,2^{\prime}, 7^{\prime}\right.$-tetrachloro-6-carboxyfluorescein) on the reverse primer.

After denaturing and renaturing the normal and mutant alleles in a mixture, mismatch heteroduplexes will be formed approximately $50 \%$ of the time. For each base change, two mismatches are formed. CEL I cuts one strand of DNA per duplex, and the digestion products are analyzed by gel electrophoresis. Data is collected electronically and viewed using Genescan ${ }^{\circledR}$ software (PE Biosystems, Foster City, CA, USA), which shows FAM as blue and TET as green. For each mismatch duplex, the assay will produce two digestion products that independently pinpoint mis- match location, making the CEL I mutation detection method highly reliable.

In this report, we show that buffers, salts, enzyme concentration and incubation time all have little impact on the CEL I mutation detection method. We further streamlined the procedure by eliminating the need for purification of the PCR product, which we previously found necessary. As a test of the efficacy of this procedure, we show (i) the evaluation of all 22 coding exons of the $B R C A 1$ gene from 10 people for the presence of mutations and/or polymorphisms (5) and (ii) the screening of 100 people for mutations and polymorphisms in a region of BRCAl using one DNA sequencing gel and multiplexed samples.

CEL I was purified from celery to near homogeneity as previously described (6). PCR primers were made in the Fannie E. Rippel Biotechnology Facility in our institution. As part of a Fox Chase Cancer Center (FCCC) Institutional Review Board-approved protocol, peripheral blood samples were obtained from consenting affected family members through the Margaret Dyson/ Family Risk Assessment Program (FRAP). Those participating in FRAP have agreed to allow their DNA to be used for a wide range of research purposes including screening for mutations in BRCAl.

Samples were prepared for enzyme digestion by PCR amplification of genomic DNA using 30 primer pairs for the coding region of the human $B R C A 1$ gene (results not shown). Each reaction was performed in a $20-\mu \mathrm{L}$ volume and amplified using AmpliTaq ${ }^{\circledR}$ DNA polymerase (PE Biosystems), using a touchdown PCR method.

CEL I $(0.2 \mathrm{U})$ was mixed with $50 \mathrm{ng}$ PCR amplification product in a $10-\mu \mathrm{L}$ reaction buffered with $3 \mathrm{mM} \mathrm{MgCl}$, $10 \mathrm{mM} \mathrm{KCl}$ and $20 \mathrm{mM}$ HEPES, $\mathrm{pH}$ 7.5. Before adding CEL I, samples were heated to $94^{\circ} \mathrm{C}$ for $1 \mathrm{~min}$ and slowly cooled to room temperature to form heteroduplexes. (1 U CEL I is defined as $1 / 1000$ of $1 \mathrm{U}$ single-stranded nuclease activity. $1 \mathrm{U}$ single-stranded DNase activity is defined as the amount of enzyme that produce $1 \mu \mathrm{g}$ acid-soluble material at $\mathrm{pH} 5.5$ in $\min 37^{\circ} \mathrm{C}$ in the absence of magnesium when purified sheared single-stranded calf thy- 